\title{
Capillary Electrophoresis in Forensic Toxicology
}

\author{
Olivier Plaut* and Christian Staub
}

\begin{abstract}
Capillary electrophoresis (CE) is a relatively young technology that can be applied to problems of interest in forensic sciences in general and toxicology in particular. Rapid analysis, high efficiencies, small sample and reagent requirements, and economical costs are advantages that $C E$ brings to forensic scientists. On the other hand, lack of sensitivity and maturity mean that CE is not yet well established in forensic labs as an additional tool, in spite of a very broad panel of applications, from ions and small molecules to macromolecules. This paper describes basic operational theory of $\mathrm{CE}$, some instrumental considerations, and applications related to forensic toxicology.
\end{abstract}

Keywords: Capillary electrophoresis $\cdot$ Forensics $\cdot$ MECC $\cdot$ Toxicology

\section{Introduction}

At the beginning of the 1980 s, three separation science technologies converged in the development of a technique which was already in existence but which suffered from the lack of appropriate technical improvements: capillary electrophoresis. The fused silica capillaries that revolutionized GC were ideally suited as separation systems. The high-sensitivity absorbance detectors developed for HPLC were easily adaptable for small diameter columns with on-tube detection. Moreover, the broad separation modes achieved by gel electrophoresis could be automated. Combining the advantages of these three powerful separation techniques has produced a new analytical tool, providing high levels of resolution with a large spectrum of applications. Among numerous advantages, one can access the ease and speed of HPLC, without the problems of large volumes of solvent to use and to dispose of. Detection is made on-line during the analysis and requires no post-run step, in contrast to gel electrophoresis. Run durations are short, as are inter-run procedures. The tiny vol-

\footnotetext{
${ }^{*}$ Correspondence: Dr. O. Plaut

Institut universitaire de médecine légale

Unité de toxicologie

Av. de Champel 9

$\mathrm{CH}-1211$ Genève 4

Tel.: +412270256 09

Fax: +41223729653

E-Mail: Olivier.Plaut@medecine.unige.ch
}

ume of sample needed - in the order of nanoliters - is a key advantage, although it limits the sensitivity.

At the end of the 1980s, commercial instruments were available and the number of published papers increased exponentially. It was time for forensic toxicologists to evaluate this technique in order to determine if it was able to provide them help in solving their daily difficulties.

\section{Theory}

Electrophoresis is the movement of charged particles in a conductive medium, under the influence of an electric field. The current generates heat due to the Joule effect. Therefore, the use of a tube of small diameter instead of flat device provides a better way of eliminating heat through the higher surface-to-volume ratio. The internal diameter of the tube has decreased from centimeters down to $3 \mathrm{~mm}$ (Hjertén, 1967) [1] to $75 \mu \mathrm{m}$ (Jorgensen, 1981) [2]. Capillary electrophoresis (CE) was born.

\subsection{Electrophoretic Flow (EPF)}

The electric field is dependant on the voltage applied to the length of the capillary. Charged species will move toward the opposite-sign electrode. Highly charged particles will move faster, whereas ion diameter and electrolyte viscosity will slow down the movement. Cations and anions will move in opposite directions and neutral species will not move.

\subsection{Electroosmotic Flow (EOF)}

Most solid surfaces in aqueous conditions exhibit excess negative charge. This is particularly the case for silica capillaries which have their silanol groups dissociated at $\mathrm{pH}$ above 3 . This creates a double layer of positive charges, one considered as 'fixed' at the walls and the other one moving toward the negatively charged electrode if an electric field is applied. These charges are solvated in the electrolyte and draw it with them. The electrolyte then moves in the tube at a velocity which depends mainly on the $\mathrm{pH}$. The characteristic of the advancing profile is flat, whereas it is parabolic in chromatography. This is one of the reasons that allow high efficiencies in CE.

The resulting movement is the combination of these two flows. As the velocity vector of the EOF is generally higher than the one of the EPF, all the species move in the same direction, in the following order: small cations, large cations, neutral species (not separated), large anions, and small anions. This basic operation mode is called capillary zone electrophoresis (CZE).

The advantage of EOF over flat electrophoresis is the ability to analyze in one step cations and anions, and to separate ions with different charge/radius ratios within a short time.

The drawback of the non-separation of neutral species can be overcome by 
changing the $\mathrm{pH}$ of the electrolyte in order to obtain charged species or by using another operating mode known as Micellar Electrokinetic Capillary Chromatography (MECC) which will be discussed later in this paper.

From a forensic point of view, CZE is a separation method based on the charge/ radius ratio that is different from the hydrophobicity generally used in chromatography. CZE is therefore known as an 'orthogonal' separation method and can be used for confirmation purpose.

\subsection{Operation Modes}

In CZE, separation is effected according to the mobility of each molecule. Main parameters influencing the EOF are voltage and temperature (which will increase EOF and efficiency while decreasing migration times), $\mathrm{pH}$, and ionic strength.

Isoelectric focusing (IEF) and isotachophoresis (ITP) will not be discussed here, as they are not widely used and have few forensic applications.

Capillary electrochromatography (CEC) is an hybrid product of CE and HPLC, using packed columns and high pressure to condition columns and inject samples, whereas the electric field is used to displace the mobile phase. In spite of numerous advantages due to the use of the best of HPLC and CE, few applications have been developed [3].

\section{Instrumentation}

A basic CE system consists of a fusedsilica capillary that connects two buffer reservoirs, two electrodes, a controllable high-voltage power supply, and a detector.

Modern systems include a pressure (or vacuum) device to rinse the capillary and to inject the sample. Carrousel autosamplers, which may be thermostated, allow automated analyses and sometimes fractions collection. The capillary has to be thermostated to yield reproducible results. The power supply generally permits a choice between constant voltage, current or power. Detectors will be discussed below. The first commercial systems were available in 1988.

\subsection{Injection}

Most suppliers provide automated injection devices, with hydrodynamic and electrokinetic injection modes.

Hydrodynamic injection is made by inserting the capillary end in the sample and applying a positive pressure to the vial or vacuum at the other end. The sample volume injected depends on time, pressure difference, capillary length and diameter, and buffer viscosity. Before automated systems became available, samples were injected by simply raising the vial to a known height for a certain time. Typically, 1 to $10 \mathrm{nl}$ of sample are injected.

Electrokinetic injection is performed by inserting the capillary end in the sample vial and applying a voltage. It allows the sample to enter the capillary by both electroosmotic and electrophoretic movement. Thus, the injected quantity depends on the electrophoretic mobility of each solute. As in CZE, small and highly charged cations move more rapidly and will be loaded to a greater extent than other species. Variations in sample conductivity due to matrix effects can affect diversely the induced current, making this mode of injection less reproducible. In addition, the discrimination for ionic species modifies the sample composition, allowing only one injection per vial. Although drawbacks may appear important with this technique, it must be mentioned that a recent development allows up to a 1000-fold improvement in peak area. This technique called 'fieldamplified on-column sample concentration' uses long injected water plugs to effect an enhanced field during electrokinetic injection of samples at negative polarity [4][5]. Parameter optimization seems to be difficult and not applicable to every solute but progress has opened a window for the analysis of toxicologically relevant substances which may be directly injected from untreated biological samples.

\subsection{Column Technology}

Most of the capillaries used are made out of fused silica. As it is easily broken, an outer layer of polyimide is placed around the capillary to strengthen it, similar to GC capillary columns. An optical window can be made by removing the polyimide coating on a short section, by burning it off with a lighter or any more sophisticated device. Pyrex capillaries are stronger than fused silica and they do not need any coating so that their entire length may be used as a window, but they are not as transparent as fused silica at wavelengths shorter than $280 \mathrm{~nm}$. There are also transparent Teflon capillaries but these are expensive and cannot be used at high voltages due to their low thermal conductivity. Untreated fused silica capillaries may be bought in bulk quantities and are therefore very cheap. However, in order to gain reproducibility with the
EOF, suppliers propose treated capillaries that have deactivated silica surfaces by chemical modification.

\subsection{Detection}

Detectors in CE have been adapted from existing HPLC systems. Therefore, the most common detectors are UV/Vis, fluorescence, electrochemical, and MS. For light-based detectors, the capillary offers the possibility to read on-line by removing the polyimide coating at the location of the light source. Although detectable masses are within the fg range, detectable concentrations remain relatively high, due to the narrow path length (25-100 $\mu \mathrm{m})$. Special detection lenses with extended path length and of course, laser-induced fluorescence (LIF) detectors enable higher sensitivity. In the forensic, non-toxicological applications of LIF-CE, we have to mention DNA sequencing. The majority of $\mathrm{CE}$ systems that are bought today are dedicated to this purpose, which is described in another paper in the present issue by our forensic biology colleagues Gehrig and Teyssier [6].

CE/MS coupling offers almost the same advantages and drawbacks as LC/ MS. The higher specificity is counterbalanced by separation problems because non-volatile mobile phases such as borate and phosphate cannot be utilized. However, the current passion for hyphenated techniques has also led to the creation of CE/MS which has made remarkable progress and a large number of papers published nowadays are devoted to such applications [7][8].

\section{Electrolyte Systems}

A wide variety of electrolyte systems are used in CE in order to perform the required separation. Most of the electrolytes are aqueous but a non-aqueous mobile phase may be interesting, for example in case of very hydrophobic analytes [9].

\subsection{CZE}

Many of the electrolytes used are similar to those used in HPLC, and are determined by the required $\mathrm{pH}$. Mobile phases are generally a mixture of phosphate, acetate, borate or zwitterionic buffers like MES or Tris.

The addition of organic modifiers (such as methanol, 2-propanol, acetonitrile) will induce complex changes when acting on electrolyte viscosity, dielectric constant, zeta potential (defined as the potential 
difference at the double layer on the capillary walls), but also on solute solvation, which can modify the apparent ionic radius.

\subsection{Micellar Electrolytes}

In 1984, Terabe et al. pushed CE a step forward by introducing Micellar Electrokinetic Capillary Chromatography (MEKC or MECC) [10], which offers separations that resemble reversephase liquid chromatography with the benefits of CE. Micelles are roughly spherical aggregates of surfactant molecules which form when their concentration is above a known critical micelle concentration. The most widely used surfactant is sodium dodecyl sulfate (SDS) which forms micelles of 62 molecules above $8 \mathrm{mM}$. SDS is available at high purity and low cost and is transparent to UV. It is an anionic surfactant, so that it forms micelles with hydrophobic tails oriented toward the center to avoid interaction with the hydrophilic aqueous buffer, with the charged heads oriented toward the buffer. SDS will migrate in the direction of the anode (positively charged electrode), that is in the opposite direction of the EOF. Since the EOF is generally faster than the migration velocity of the micelles, the net movement will be in the direction of the cathode. Solutes will interact with the micelles through hydrophobic and electrostatic interactions. The more the solute interacts with the micelle the longer its migration time. Benefits of MECC include separation of neutral species, solubilization of hydrophobic molecules, including proteins that could interact irreversibly with the capillary walls. This opens a large window for the analysis of biological fluids.

\subsection{Other Electrolytes}

Many other 'exotic' mixtures have been used in CE but few have real applications in the forensic field, except cyclodextrins, which will be described in the following chapter.

\section{Applications}

The are numerous applications in the field of forensic toxicology. Some recently published reviews give an actual progress report on the question [11][12]. During the first decade of its exponential development, authors tried to demonstrate that CE was able to perform any task as well as other analytical techniques. This objective was only partially reached and only a tiny number of foren- sic labs have today $\mathrm{CE}$ instruments performing routine tasks, whereas $\mathrm{CE}$ has its place in other applications, like quality control in pharmaceutical analysis [13].

Extensive reviews of clinical and forensic applications are available in a symposium report by Caslavska and Thormann [14], and in a recent book edited by Petersen and Mohammad [15]. Therefore, instead of giving a long list of analytes and the corresponding reference, we are going to give below only some examples where $\mathrm{CE}$ exhibits various advantages over conventional chromatographic techniques.

\subsection{Analysis of Non-biological Samples}

$\mathrm{CE}$ is well-suited for the general field of drugs-of-abuse seizures. Sensitivity is not a problem, analyses can be automated, and derivatization is not required for the determination of compounds that would require derivatization before $\mathrm{GC}$ analysis, like morphine and benzoylecgonine. The electropherogram shown on Fig. 1 is a particular application in forensic sciences: an almost empty syringe was found from which $5 \mu$ of liquid could be extracted. This sample was injected in a CE systems without any preparation. As the injected volume was below $10 \mathrm{nl}$, the unmodified sample could be used for other analyses or conservation. Peak identification was performed with UV spectra. As for other techniques, it is of great importance that spectra libraries exist and can be used by any equipment, a condition that has not yet been understood by some manufac- turers. In the case of an unknown mixture, a real advantage of CE is the possibility to inject aqueous samples without the fear of damaging an expensive column. Worn capillaries may be exchanged for less than $10 \mathrm{CHF}$.

Amphetamines, including Ecstasylike amphetamines, may be analyzed by CZE in a short time and with high efficiency [16]. The addition of cyclodextrins $(\mathrm{CD})$ to the electrolyte allows the separation of chiral enantiomers [17]. CDs are barrel-shaped macrocyclic oligosaccharides with highly hydrophobic, optically active cores. The very small volume of electrolyte used in $\mathrm{CE}$ authorizes the use of expensive additives like $\mathrm{CD}$, whereas in HPLC chiral groups have to be fixed on the stationary phase.

Non-steroidal anti-inflammatory drugs (NSAID) are often difficult to analyze by chromatographic methods, due to their acidic properties. As their dosage is generally high, they are well suited for CE analysis. Fig. 2 shows the separation of 7 common NSAID in $4 \mathrm{~min}$.

\subsection{Analysis of Biological Samples}

A permanent challenge for forensic toxicologists is the 'general unknown screening'. This procedure relies for a large part on spectra libraries, available for UV and MS detectors. Some UV libraries are compatible with some CE systems, but not all of them. Hudson and coworkers [18] developed a comprehensive screen for drugs in whole blood, using CZE. They published a list of several hundreds of compounds, to be identified by migration time and UV spectra, and

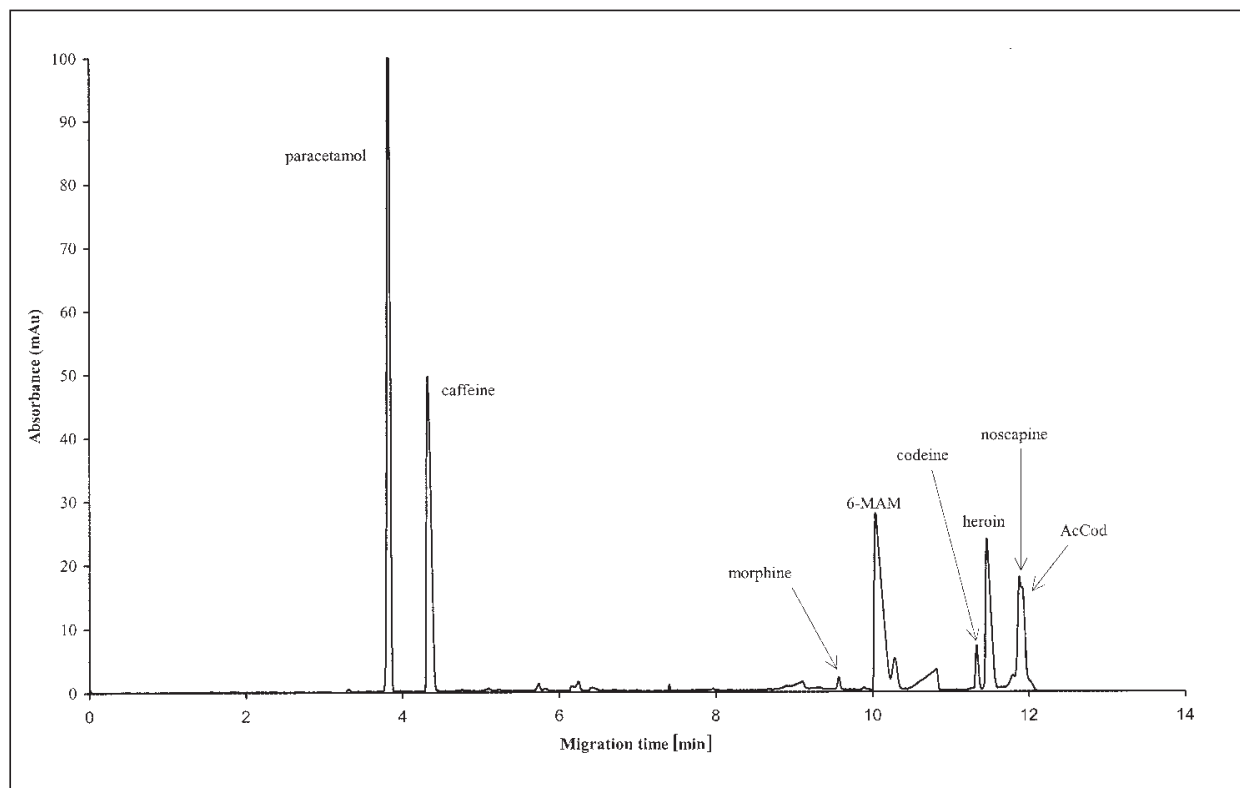

Fig. 1. Electropherogram of a syringe content. Capillary: $50 \mathrm{~cm} \times 50 \mu \mathrm{m}$; voltage: $20 \mathrm{kV}$; buffer: borate/phosphate, $\mathrm{pH} 8.2$, SDS $50 \mathrm{mM}$; capillary temperature: $30^{\circ} \mathrm{C}$; acquisition: $195 \mathrm{~nm}$; CE System: Biofocus 3000 , Bio-Rad Laboratories, Hercules, CA, USA. 


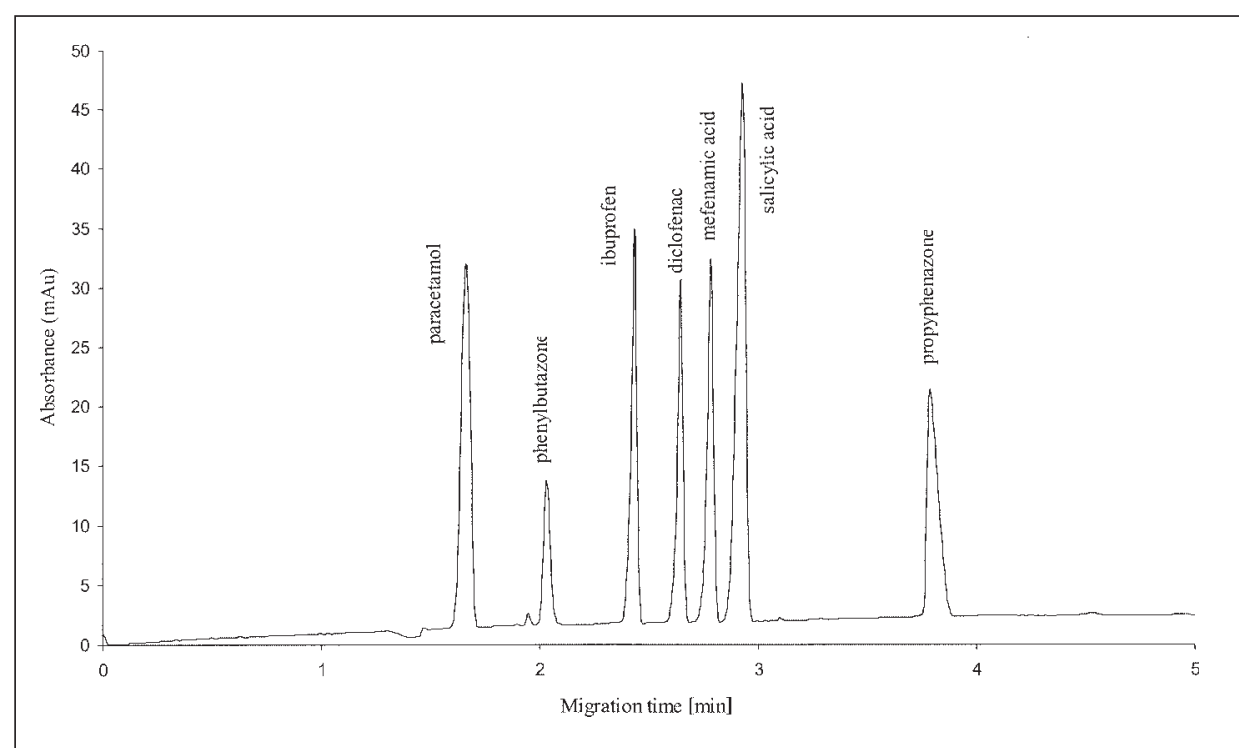

Fig. 2. Separation of 7 NSAID. Concentration of each analyte: $50 \mu \mathrm{g} / \mathrm{ml}$; capillary: $36 \mathrm{~cm} \times 50 \mu \mathrm{m}$. Otherwise same conditions as in Fig. 1.

also by apparent mobility that can be calculated by the CE system. The mobility calculation offers a more reproducible way to identify peaks.

Most forensic analyses of unknown cases start with immunoassay. Although it is not yet well developed, we have to mention that CE can be used to perform immunoassays in a very spectacular way, due to the tiny quantities used: as the vol- ume of the mobile phase does not exceed a few microliters, Regnier and coworkers [19] used the sample as a mobile phase and injected the enzymatic reagent as if it was the sample! The reagent then reacts with the analytes and is ready to be quantitatively analyzed by the time it reaches the detector window.

Urine, serum, saliva, vitreous humor, and cerebro-spinal fluid are liquids that

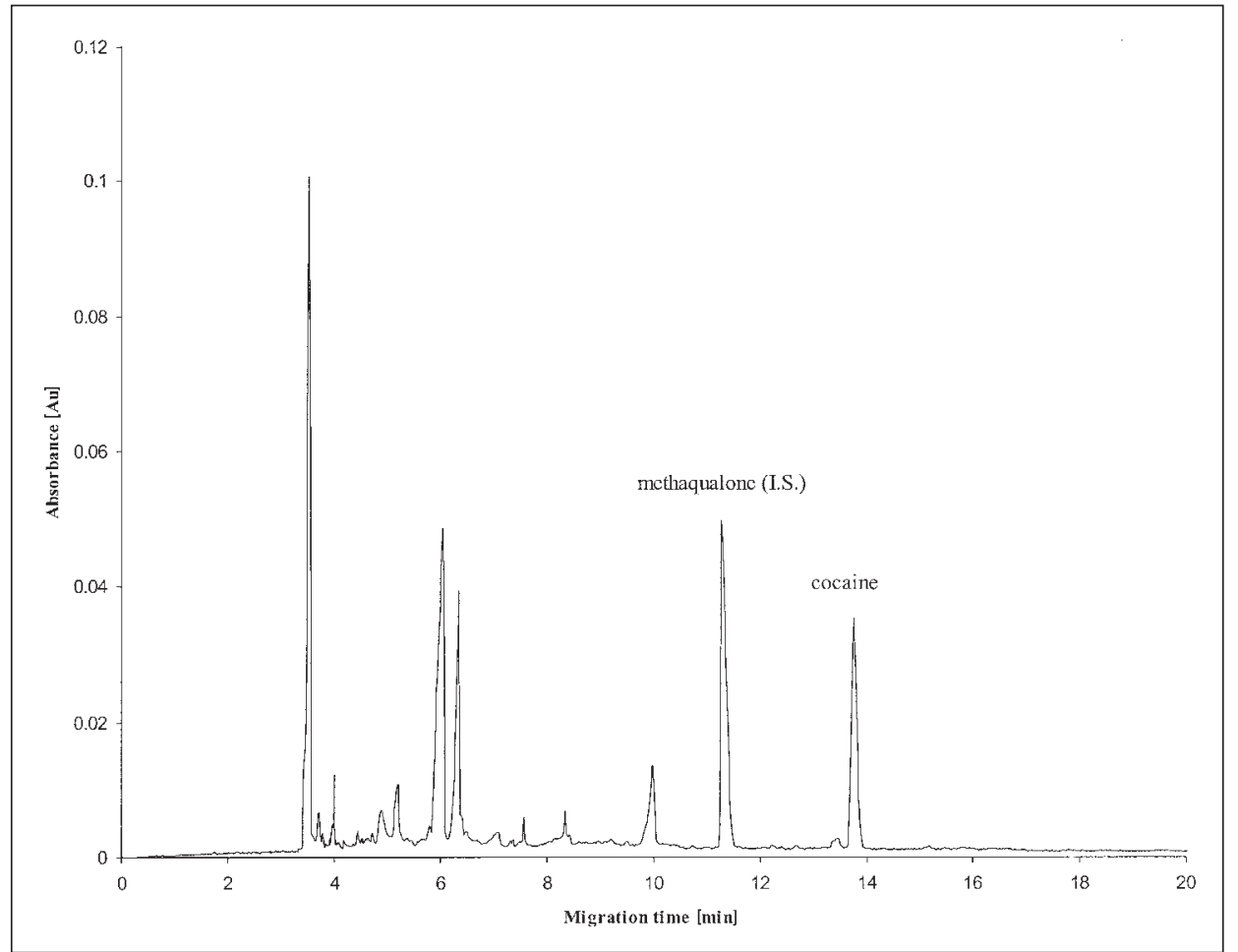

Fig. 3. Electropherogram of a urine sample, containing methaqualone as internal standard. Buffer: borate/phosphate, pH 8.75; acquisition: 200 nm. Otherwise same conditions as in Fig. 1. can be injected directly in $\mathrm{CE}$, but this type of analysis will generally face a lack of sensitivity, except in cases of massive intoxication. Fig. 3 shows the direct injection of an urine sample from a man who ingested cocaine bundles in order to cross customs (body-packer). The man was taken to hospital for surgery after one bundle cracked in his intestine.

Gastric liquid, after centrifugation, is well suited for $\mathrm{CE}$ analysis. Fig. 4 shows the analysis of gastric contents after intoxication by atropine, strychnine, and tetracaine. Panel A shows the electropherogram obtained after direct injection and panel B the electropherogram obtained after extraction.

Differences in migration times are due to the matrix effect: the different ionic strength of the sample plug modifies the apparent conductivity of the electrolyte as long as the plug is in the capillary. Atropine is difficult to analyze: It decomposes in GC injectors and yields bad peak shapes in HPLC. This is why it is generally analyzed by immunoassay and thinlayer chromatography. CE is therefore well suited for the analysis of atropine and other tropane alkaloids [20].

Carbohydrate-deficient transferrin (CDT) is a marker of chronic alcohol abuse, which is of forensic interest in cases of reissuing driving licenses. CDT indicates a group of isoforms of human transferrin (Tf), the main iron transport serum protein, deficient in sialic acid residues (asialo-, monosialo- and disialo-Tf) in comparison to the main isotransferrin which contains four sialic acid groups (tetrasialo-Tf). Some available immunoassays suffer from interference which may conduct to false positive results by cross-reaction with trisialo-Tf. CE is a method suitable for confirmation of CDT immunoassays by an independent technique [21].

Quaternary ammonium ions are generally difficult to analyze. This is the case for curare-like compounds and also herbicides, like paraquat, diquat, and difenzoquat. Those three compounds can be successfully analyzed by CE, not only in cases of intoxication [22] but also to determine residues in agriculture.

Although it is not strictly a forensic application, the binding between drugs and proteins is of interest for forensic scientists and can be studied by CE. Affinity capillary electrophoresis (ACE) permits noncovalent interactions of macromolecules with ligands to be evaluated, which create mobility changes when they bind together [23]. 


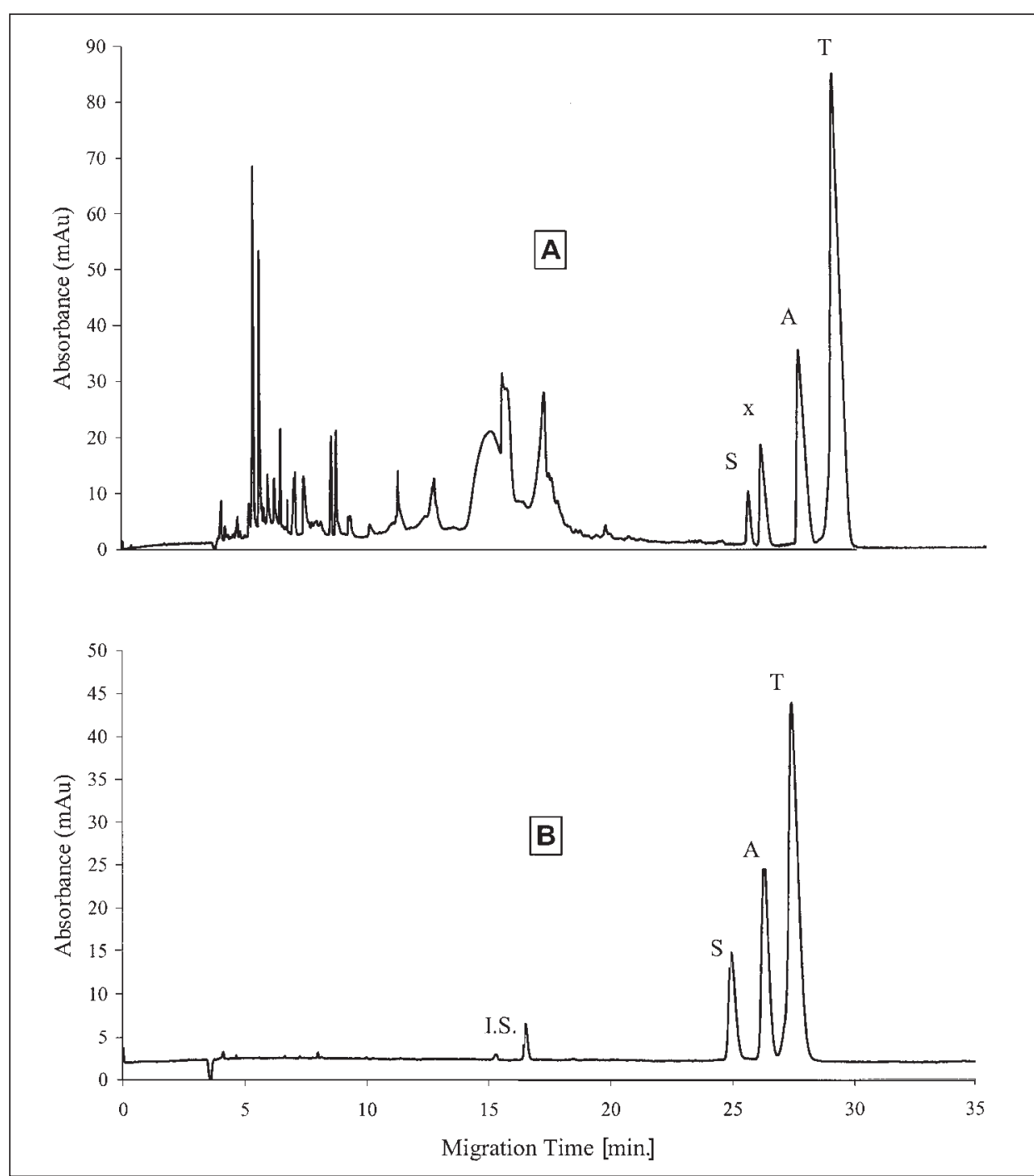

Fig. 4. Electropherogram of a gastric-content sample, after direct injection (A) and after liquidliquid extraction at $\mathrm{pH} 9$ with butyl chloride, using scopolamine as internal standard (B). Buffer: borate/phosphate, pH 9.2. Otherwise same conditions as in Fig. 1.

\section{Conclusion}

$\mathrm{CE}$ is a versatile technique that offers several advantages which earns it a place in the forensic lab. Tiny sample volumes, low reagent consumption, automation, low-cost capillaries are arguments that everyone is aware of today. On the other hand, this rather young technique has generally a low sensitivity and a bad reproducibility, if compared to chromatographic methods. UV spectra are often not specific enough, when looking for absolute identification. Spectra libraries should be more widely available and every system should have an automated identification software, but this is not yet the case. In fact, after a very promising start and exponential development, the enthusiasm for CE seems to have reached a plateau and manufacturers are no longer making large efforts to improve their systems. The future of CE may well be
[1] S. Hjerten, Chromatogr. Rev. 1967, 9, 122.

[2] J.W. Jorgenson, K.D. Lukacs, Anal. Chem. 1981, 53, 1298.

[3] J.H. Miyawa, M.S. Alesandro, $L C$-GC Int . 1998, 16, 36.

[4] R.L. Chien, D.S. Burgi, Anal. Chem. 1992, 64, 489A

[5] C.X. Zhang, W. Thormann, Anal. Chem. 1996, 68, 2523.

[6] C. Gehrig, A. Teyssier, Chimia, 2002, 56, 71.

[7] J.F. Banks, Electrophoresis 1997, 18 , 2255.

[8] S. Cherkaoui, S. Rudaz, E. Varesio, J.L. Veuthey, Chimia 1999, 53, 501.

[9] S. Cherkaoui, L. Mateus, P. Christen, J.L. Veuthey, Chromatographia 1999, 49, 54.

[10] S. Terabe, K. Otsuka, K. Ichikawa, A. Tsuchiya, T. Ando, Anal. Chem. 1984, 56, 111.

[11] F. Tagliaro, S. Turrina, P. Pisi, F.P. Smith, M. Marigo, J. Chromatogr. B 1998, 713, 27.

[12] N.P. Lemos, F. Bortolotti, G. Manetto, R.A. Anderson, F. Cittadini, F. Tagliaro, Sci. Justice 2001, 41, 203.

[13] K.D. Altria, A.B. Chen, L. Clohs, $L C-G C$ Europe 2001, 14, 736.

[14] J. Caslavska, W. Thormann, Eds., Electrophoresis 1998, 19(16-17).

[15] J.R. Petersen, A.A. Mohammad, 'Clinical and forensic applications of capillary electrophoresis', Humana Press, Totowa N.J., 2001.

[16] Y. McAvoy, M.D. Cole, O. Guéniat, For. Sci. Int. 1999, 102, 13.

[17] E. Varesio, J.L. Veuthey, J. Chromatogr. A 1995, 717, 219.

[18] J.C. Hudson, M. Golin, M. Malcolm, Can Soc. Forensic Sci. J. 1995, 28, 137.

[19] J. Bao, F.E. Regnier, J. Chromatogr. 1992, 608, 217.

[20] O. Plaut, C. Staub, Electrophoresis 1998, 19, 3003.

[21] F. Tagliaro, F. Bortolotti, M. Zuliani, F. Crivellente, G. Manetto, V.L. Pascali, M. Marigo, J. Capillary Electrophor. Michrochip Technol. 1999, 6, 137.

[22] M.T. Galceran, M.C. Carneiro, L. Puignou, Chromatographia 1994, 39, 581.

[23] N.H. Heegaard, S. Nilsson, N.A. Guzman, J. Chromatogr. B Biomed. Appl. 1998, 715, 29.

[24] L.J. Kricka, Clin. Chim. Acta 2001, 307, 219.

has a role to play here. For example, sample preconcentration by field amplification, which has been described in the injection chapter, has been applied to microchip-based CE in Neuchâtel by de Rooij and coworkers [25].

As mass spectrometry coupling with liquid systems becomes more widely available, CE/MS allows for sensitive and specific determinations in biological samples, either with atmospheric pressure ionization [26] or with electrospray ionization [27] and could retain its (small) role as an alternate tool for $\mathrm{LC} /$ MS users.

Received: January 29, 2002
[25] J. Lichtenberg, E. Verpoorte, N.F. de Rooij, Electrophoresis 2001, 22, 258.

[26] G. Hopfgartner, M. Zell, C. Husser, A. Maschka-Selig, B. Lausecker, Chimia 1999, 53, 469.

[27] S. McClean, E. O'Kane, W.F. Smyth, J. Chromatogr. A 1999, 838, 273. 